\title{
Development of an isothermal amplification-based assay for the rapid detection of Cronobacter spp.
}

\author{
Siying Liu, ${ }^{* 1}$ Yunyun Geng, ${ }^{*} \dagger^{1}$ Libing Liu, $¥ \S$ Xiaoxia Sun, $¥ \S$ Jingyu Shao, ${ }^{*}$ Beibei Han, ${ }^{*}$ Jianchang Wang, $¥ \S^{2}$ \\ and Ke Tan*2 \\ *Key Laboratory of Animal Physiology, Biochemistry and Molecular Biology of Hebei Province, College of Life Sciences, Hebei Normal University, \\ Shijiazhuang, Hebei 050024, China \\ †College of Basic Medicine, Hebei University of Chinese Medicine, Shijiazhuang, Hebei 050020, China \\ $\ddagger$ Center of Inspection and Quarantine, Hebei Entry-Exit Inspection and Quarantine Bureau, Shijiazhuang, Hebei 050051, China \\ $\S$ Hebei Academy of Inspection and Quarantine Science and Technology, Shijiazhuang, Hebei 050051, China
}

\begin{abstract}
Cronobacter spp. is an opportunistic pathogen that is associated with rare but life-threatening neonatal infections resulting from the consumption of contaminated powdered infant formula milk (PIF). In the present study, we developed recombinase polymerase amplification (RPA) and real-time RPA for the detection of Cronobacter spp. in PIF for the first time by targeting the ompA gene. The specificity and sensitivity of the RPA and real-time RPA were validated and the practical applicability of these methods for the detection of Cronobacter spp. in artificially contaminated PIF samples was proved by comparing their reaction time, sensitivity, and efficacy with those of real-time PCR and the Chinese traditional method. The RPA and real-time RPA assays reduced the analysis time to less than $15 \mathrm{~min}$ and the results were as reliable as those of real-time PCR. Taken together, the RPA and realtime RPA assays served as fast, reliable, and sensitive techniques for the detection of Cronobacter spp.
\end{abstract}

Key words: Cronobacter spp., recombinase polymerase amplification, real-time recombinase polymerase amplification, real-time PCR

\section{INTRODUCTION}

Cronobacter spp. (formerly Enterobacter sakazakii) is an opportunistic pathogen associated with sporadic outbreaks of severe meningitis, sepsis, and necrotizing enterocolitis in neonates and infants with a high mortality rate of 33 to $80 \%$ (Willis and Robinson, 1988; van Acker et al., 2001; Hunter and Bean, 2013). This

\footnotetext{
Received October 3, 2017.

Accepted January 16, 2018.

${ }^{1}$ These authors contributed equally to this article.

${ }^{2}$ Corresponding authors: jianchangwang1225@126.com and tanke0331@126.com
}

genus of bacteria consists of 7 species: Cronobacter sakazakii, Cronobacter dublinensis, Cronobacter malonaticus, Cronobacter muytjensii, Cronobacter turicensis, Cronobacter universalis, and Cronobacter condimenti. A growing number of reports have noted that Cronobacter spp. could be isolated from a variety of foods, and powered infant formula milk (PIF) was identified as a major source of Cronobacter spp. Infections (Muytjens et al., 1988; Drudy et al., 2006; Pina-Pérez et al., 2016). It is currently thought that even very low levels of Cronobacter spp. in PIF (0.36-0.66 cfu/100 g) can lead to infections in neonates (Muytjens et al., 1988; Drudy et al., 2006; Pina-Pérez et al., 2016). As the symptoms of the infection are severe, the prognosis is poor, and even low numbers of Cronobacter spp. pose a health risk, development of a rapid and sensitive technique for early detection of Cronobacter spp. in foods is of utmost importance.

To reduce or prevent the hazards posed by Cronobacter spp., a great deal of effort has been invested in the development of methods to eliminate Cronobacter spp. infection. The traditional biochemical procedures recommended by the US Food and Drug Administration (FDA) are laborious and time-consuming (FDA, 2002). To date, a variety of molecular detection and diagnostic techniques for Cronobacter spp. have been developed. The use of molecular methods, such as PCR, real-time PCR, loop-mediated isothermal amplification, and PCR-ELISA, has facilitated pathogen detection (Park et al., 2012; Cai et al., 2013; Shukla et al., 2016). Both PCR and real-time PCR assays have been increasingly exploited and characterized by a wide dynamic range of quantification, high sensitivity, and a high degree of precision (Hu et al., 2016; Li et al., 2016; Chen et al., 2017). Despite these improvements, these molecular methods are very complicated in terms of sample pretreatment and measuring procedures, are time-consuming, and require expensive laboratory apparatus and experienced technicians (Qiming et al., 
2015; Zhou et al., 2016; Yu et al., 2017). Recently, the recombinase polymerase amplification (RPA) assay, an isothermal gene amplification method, has been developed as a potential strategy for various pathogens detection (Lillis et al., 2016; Wang et al., 2017a,b). The RPA assay not only is sensitive and specific but also has the advantages of rapidity and simplicity (Lillis et al., 2016; Abd El Wahed et al., 2015a,b).

In the present study, we developed RPA and realtime RPA for the detection of Cronobacter spp. in PIF for the first time by targeting the ompA gene. Our newly developed RPA and real-time RPA assays could be successfully used for sensitive and specific detection of Cronobacter spp. in contaminated PIF samples with a significantly reduced time consumption. The analysis time was reduced to less than $15 \mathrm{~min}$, and our assays were as reliable as real-time PCR and the standard method GB4789.40-2016 (CFDA, 2016). Therefore, the RPA and real-time RPA assays serve as fast, reliable, and sensitive techniques for the detection of Cronobacter spp.

\section{MATERIALS AND METHODS}

\section{Bacterial Stains}

A total of 47 common pathogenic bacteria associated with dairy products and human health are shown in Table 1. The sources of these strains are listed. All strains were cultured in tryptic soy broth at $37^{\circ} \mathrm{C}$.

\section{Generation of Standard DNA}

Bacterial genomic DNA was isolated using a TIANamp Bacterial DNA kit (Tiangen, Beijing, China) according to the manufacturer's instruction (http://www.tiangen .com/asset/imsupload/up0994144001433140765.pdf). The concentration of bacterial genomic DNA was quantified using a ND-2000c spectrophotometer (NanoDrop, Wilmington, DE). The copy number of DNA molecules was calculated by the formula amount $($ copies $/ \mu \mathrm{L})=$ [DNA concentration $(\mathrm{g} / \mu \mathrm{L}) /($ bacterial genome length in base pairs $\times 660)] \times 6.02 \times 10^{23}$.

\section{Primers and Exo Probe}

Nucleotide sequences of Cronobacter spp. were aligned to identify conserved regions of the ompA gene. Primers and exo probes were designed based on the conserved region of the ompA gene using the Primer 5.0 software (Premier Biosoft International, Palo Alto, CA), as described in Table 2. The real-time RPA primers and probes were selected by testing the combination to yield the highest sensitivity. Primers and exo probes were synthesized by a commercial company (Sangon Biotech, Shanghai, China).

\section{RPA and Real-Time RPA Assay}

The RPA and real-time RPA reactions were performed in a $50 \mu \mathrm{L}$ volume using a TwistAmp basic kit (TwistDX, Cambridge, UK) and TwistAmp exo kit (TwistDX), respectively. Other components included $420 \mathrm{n} M$ aliquots of each RPA primer, $14 \mathrm{~m} M$ magnesium acetate, and $1 \mu \mathrm{L}$ of bacterial DNA. For the real-time RPA assay, $120 \mathrm{n} M$ exo probe was also added to the mixture. The reaction tubes were placed in a Genie III scanner device (OptiGene Limited, West Sussex, UK) to start the reaction at $38^{\circ} \mathrm{C}$. The fluorescence signal was recorded in real-time and increased markedly upon successful amplification.

\section{Real-Time PCR}

Real-time PCR was performed using ABI 7500 (Applied Biosystems, Foster City, CA) instrument. The sequences of the primers and probe are listed in Table 2. The Premix Ex Taq (Takara Co. Ltd., Dalian, China) was applied for real-time PCR, and the reaction was performed at $95^{\circ} \mathrm{C}$ for $30 \mathrm{~s}$, followed by 35 cycles of $94^{\circ} \mathrm{C}$ for $5 \mathrm{~s}$ and $60^{\circ} \mathrm{C}$ for $34 \mathrm{~s}$.

\section{Analytical Specificity and Sensitivity Analysis}

The analytical specificity was evaluated against a panel of pathogenic bacteria considered to be important in food (Table 1). To analyze the RPA sensitivity, genomic DNA from Cronobacter sakazakii (ATCC29544) was 10-fold serially diluted to achieve DNA concentrations ranging from $10^{7}$ to $10^{0}$ copies/ $\mu \mathrm{L}$. One microliter of each DNA dilution was used as a template and amplified with the RPA or real-time RPA assay. Real-time RPA was tested using the standard DNA in 8 replicates. The threshold time was plotted against the molecules detected.

\section{PIF Spiking with Cronobacter spp.}

Commercially available PIF was used to certify the potential use and the suitability of RPA assay. The PIF products were obtained from supermarkets in China. The absence of Cronobacter spp. was tested in PIF using the National Food Safety Standard of Enterobacter sakazakii Examination (GB4748.40-2016) of China (CFDA, 2016). One hundred grams of PIF and 10, 50, and $100 \mathrm{cfu}$ of Cronobacter spp. were added to peptone water and then incubated for 6 or $8 \mathrm{~h}$ at $37^{\circ} \mathrm{C}$. One milliliter of each bacterial culture was centrifuged 
Table 1. Bacterial strains and analytical specificity results for recombinase polymerase amplification (RPA) and real-time RPA assays

\begin{tabular}{|c|c|c|c|}
\hline Strain name & Origin $^{1}$ & $\mathrm{RPA}^{2}$ & Real-time $\mathrm{RPA}^{2}$ \\
\hline Cronobacter sakazakii & ATCC 29544 & + & + \\
\hline Cronobacter sakazakii & ATCC 29004 & + & + \\
\hline Cronobacter sakazakii & ATCC 12868 & + & + \\
\hline Cronobacter sakazakii & CICC 21560 & + & + \\
\hline Cronobacter muytjensii & ATCC 51329 & + & + \\
\hline Cronobacter muytjensii & CICC 21563 & + & + \\
\hline Cronobacter malonaticus & CICC 21551 & + & + \\
\hline Cronobacter turicensis & CICC 24178 & + & + \\
\hline Cronobacter dublinensis & CICC 21564 & + & + \\
\hline Cronobacter universalis & CICC 21570 & + & + \\
\hline Campylobacter jejuni & ATCC 33291 & - & - \\
\hline Citrobacter freundii & ATCC 10787 & - & - \\
\hline Enterococcus faecalis & ATCC 29212 & - & - \\
\hline Listeria monocytogenes & ATCC 19114 & - & - \\
\hline Klebsiella pneumoniae & ATCC 4352 & - & - \\
\hline Proteus mirabilis & ATCC 29906 & - & - \\
\hline Providencia & ATCC 29944 & - & - \\
\hline Serratia marcescens & ATCC 14756 & - & - \\
\hline Staphylococcus aureus & ATCC 6538 & - & - \\
\hline Bacillus cereus & CICC 10648 & - & - \\
\hline Escherichia coli O157:H7 & CICC 21530 & - & - \\
\hline Salmonella Typhimurium & CICC 22956 & - & - \\
\hline Shigella flexneri & CICC 21678 & - & - \\
\hline Shigella sonnei & CICC 21679 & - & - \\
\hline Vibrio parahaemolyticus & CICC 21617 & - & - \\
\hline Yersinia enterocolitica & CICC 21609 & - & - \\
\hline Bacillus cereus & CMCC 63301 & - & - \\
\hline Klebsiella pneumoniae & CMCC 46117 & - & - \\
\hline Proteus mirabilis & CMCC 49005 & - & - \\
\hline Salmonella Paratyphi & CMCC 50671 & - & - \\
\hline Salmonella Paratyphi & CMCC 50094 & - & - \\
\hline Salmonella spp. & CMCC 50760 & - & - \\
\hline Salmonella spp. & CMCC 50540 & - & - \\
\hline Shigella spp. & CMCC 51197 & - & - \\
\hline Shigella spp. & CMCC 51062 & - & - \\
\hline Shigella spp. & CMCC 51334 & - & - \\
\hline Staphylococcus aureus & CMCC 26003 & - & - \\
\hline Staphylococcus epidermidis & CMCC 26069 & - & - \\
\hline Staphylococcus albus & CMCC 26101 & - & - \\
\hline Staphylococcus pyogenes & CMCC 26103 & - & - \\
\hline Staphylococcus citreus & CMCC 26106 & - & - \\
\hline Streptococcus haemolyticus & CMCC 3220 & - & - \\
\hline Streptococcus haemolyticus & CMCC 32210 & - & - \\
\hline Bacillus licheniformis & Isolated by our laboratory & - & - \\
\hline Enterobacter cloacae & Isolated by our laboratory & - & - \\
\hline Mannheimia haemolytica & Isolated by our laboratory & - & - \\
\hline Pseudomonas aeruginosa & Isolated by our laboratory & - & - \\
\hline
\end{tabular}

Table 2. The sequences of primers and probes used in this study

\begin{tabular}{|c|c|}
\hline Gene & Primer and probe \\
\hline
\end{tabular}


at $10,000 \times g$ for $3 \mathrm{~min}$ at $4^{\circ} \mathrm{C}$. The supernatant was then discarded carefully and the cell pellet was washed with PBS. After centrifugation, the cell pellet was resuspended and subjected to template extraction using a Bacterial Genomic DNA Extraction Kit (Tiangen). The bacterial genomic DNA samples were stored at $-20^{\circ} \mathrm{C}$ until use.

\section{RESULTS}

Outer membrane protein A, which is essential for basolateral invasion of Cronobacter spp., was reported to be suitable for the detection of Cronobacter spp. (Cai et al., 2013). The ompA gene seems to have higher specificity than other genes, such as ITS (16S-23S rRNA internal transcribed spacer), $16 \mathrm{~S}$ rRNA, $z p x$, and gluA and gluB (Jaradat et al., 2009; Cai et al., 2013). Based on the published DNA sequences of the OmpA gene of Cronobacter spp. and the results of BLAST (https:// blast.ncbi.nlm.nih.gov/Blast.cgi) homology analysis, primers were designed using the Primer 5.0 software as described in Table 2. The primers amplified specific and significant band at $294 \mathrm{bp}$, as expected.

\section{Analytical Specificity and Sensitivity of the RPA Assay}

The analytical specificity of RPA assay was examined by using different pathogen genomic DNA as templates. As shown in Table 1, only Cronobacter spp. was detected successfully, including Cronobacter sakazakii, Cronobacter muytjensii, Cronobacter malonaticus, Cronobacter turicensis, Cronobacter dublinensis, and Cronobacter universalis, whereas other bacterial species were not detected. The results demonstrated the high specificity of the RPA assay.

The analytical sensitivity of RPA assay was determined by diluting the DNA concentrations of Cronobacter spp. from $10^{7}$ to $10^{0}$ copies/reaction. The specific 294-bp amplicon could be detected when the DNA concentration reached $10^{4}$ copies/reaction (Figure 1A). To prove that RPA is a more rapid, technically simpler detection method, we examined the reaction time of the RPA assay. As shown in Figure 1B, positive results were obtained at $38^{\circ} \mathrm{C}$ within $10 \mathrm{~min}$. The intensity of the 294-bp amplicon increased when the reaction time was up to $20 \mathrm{~min}$; however, the intensity did not further increase after $20 \mathrm{~min}$. These results indicated that the RPA assay was much faster than real-time PCR, which normally takes over $1 \mathrm{~h}$.

Recently, real-time PCR assays have been exploited and characterized by high sensitivity. Therefore, we compared the analytical sensitivity of the RPA assay with that of real-time PCR. Figure 2A shows that real-time PCR also had the same detection limit, $10^{4}$ copies/reaction, using template DNA prepared from 10-fold serial dilutions of DNA of the same Cronobacter strain. The probit regression analysis on data from 8 runs demonstrated that the limit of detection (LOD) of real-time PCR was about $4.5 \times 10^{4}$ copies/reaction in $95 \%$ of cases (Figure 2B). These results indicated that the RPA assay possessed excellent and comparable analytical sensitivity to real-time PCR.

\section{Analytical Specificity and Sensitivity of the Real-Time RPA Assay}

To further improve the advantage of RPA assay, we developed real-time RPA by designing the specific exo probe based on the RPA assay. We first examined the analytical specificity of the real-time RPA assay. As shown in Table 1, real-time RPA detected only Cronobacter spp., but not other bacterial species. The results indicated the excellent specificity of the real-time RPA assay. The analytical sensitivity of the real-time RPA assay was determined by using the same diluted DNA concentrations of Cronobacter spp. as shown in Figure 1. The detection limit of real-time RPA was $10^{4}$ copies/ reaction, the same as those of real-time $\mathrm{PCR}$ and the RPA assay (Figure 3A). These results indicated that real-time RPA possessed prominent merits of analytical sensitivity and specificity, similar to real-time PCR.

With the average reaction time from 8 runs on the DNA standards, we made a semi-log regression analysis for the real-time RPA by using GraphPad Prism 7.0 (GraphPad Software Inc., San Diego, CA). The runtimes of the real-time RPA assay were approximately 8 to 15 min for $10^{7}$ to $10^{4}$ copies (Figure $3 \mathrm{~B}$ ), whereas real-time PCR with cycle threshold values between 21 and 31 required approximately 32 to $45 \mathrm{~min}$ to obtain the final results. Moreover, a probit regression analysis using results of 8 complete molecular standard runs calculated that the LOD of real-time RPA was about $2.3 \times 10^{4}$ copies/reaction in $95 \%$ of cases (Figure 3C). These results indicated that the real-time RPA assay was much faster than but still as reliable as real-time PCR.

\section{Validation of Real-Time RPA with Artificially Contaminated PIF}

To accurately demonstrate the analytical sensitivity and high-speed of RPA and real-time RPA for detecting Cronobacter spp., PIF was contaminated artificially with different amounts of Cronobacter spp. for different enrichment times (Table 3). During enrichment procedures, the growth of other pathogens was considered an inhibitory factor for the target microbial flora, leading 
to an increase in the detection limit. The diagnostic performance of the real-time RPA assay to detect Cronobacter spp. was compared with that of the traditional method (GB4789.40-2016) and real-time PCR. The traditional method is the national food safety standard method for Cronobacter spp. examination in China. This standard stipulates the testing method of Cronobacter spp. in food and applies to the examination of Cronobacter spp. in infant formula food, dairy products, and their raw materials. The traditional method is equivalent to the procedures recommended by the US FDA and ISO (FDA, 2002; ISO, 2006). After enrichment for $8 \mathrm{~h}$, real-time RPA obtained positive results within $12.85,10.8$, or 8.18 min when PIF samples were contaminated with 10,50 , or $100 \mathrm{cfu} / 100$ g of Cronobacter spp., respectively; whereas real-time PCR with cycle threshold values at $33.68,33.12$, and 26.93 required approximately 51, 50, and $41 \mathrm{~min}$, respectively (Table 3). Using the traditional method, similar positive final results could be obtained when contaminated samples were enriched for $8 \mathrm{~h}$ (Table 3). These results demonstrated that real-time RPA had a diagnostic agreement of $100 \%$ with real-time PCR and the traditional method. However, real-time RPA was much faster than real-time PCR and the traditional method. After $6 \mathrm{~h}$ of enrichment, none of these 4 methods could detect Cronobacter spp., indicating no false-positive results from samples containing a low level of Cronobacter spp. (Table 3). Therefore, the realtime RPA method is suitable for a reliable detection of low numbers of Cronobacter spp. in connection with an appropriate enrichment time.

\section{DISCUSSION}

Cronobacter spp. has become increasingly recognized as an opportunistic pathogen associated with neonatal infections resulting from the consumption of contaminated PIF (Kandhai et al., 2004). Although rare, infections caused by Cronobacter spp. are often life-

$\mathbf{A}$

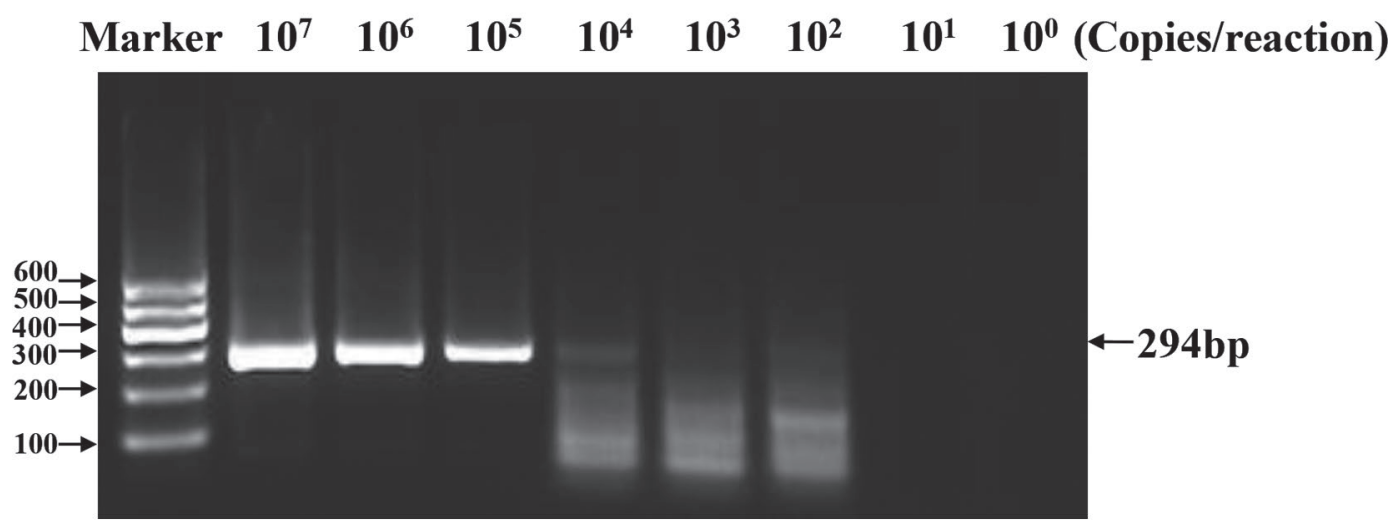

B

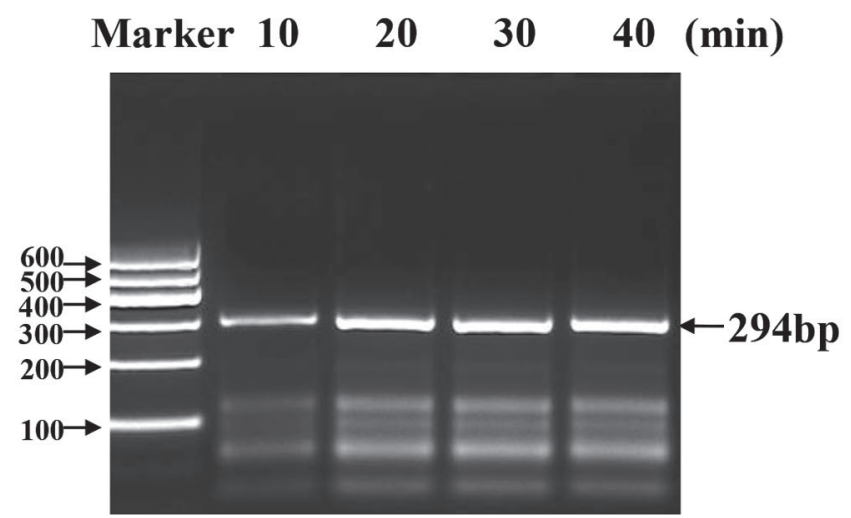

Figure 1. Analytical sensitivity and reaction time of recombinase polymerase amplification (RPA) assay. (A) The analytical sensitivity of RPA assay was determined by diluting the DNA concentrations of Cronobacter spp. from $10^{7}$ to $10^{0}$ copies/reaction. The specific 294 -bp amplicon could be detected when the DNA concentration reached $10^{4}$ copies/reaction. (B) Positive results were obtained at $38^{\circ} \mathrm{C}$ within 10 min. The intensity of 294-bp amplicon increased when the reaction time was up to 20 min. 
threatening and most frequently cause meningitis, sepsis, and necrotizing enterocolitis (Willis and Robinson, 1988; Hunter and Bean, 2013). Because even low levels of Cronobacter spp. contamination could pose a health risk, accurate and sensitive methods are required for the rapid detection of Cronobacter spp. in food (Muytjens et al., 1988; Drudy et al., 2006; Pina-Pérez et al., 2016).
Here, we developed RPA and real-time RPA assays to rapidly identify the Cronobacter spp. with high analytical sensitivity and specificity. We examined the high specificity of RPA and real-time RPA with DNA from 47 bacterial strains. Among them, only Cronobacter spp. strains were correctly amplified, whereas no positive signal was observed from 37 nontarget pathogens.

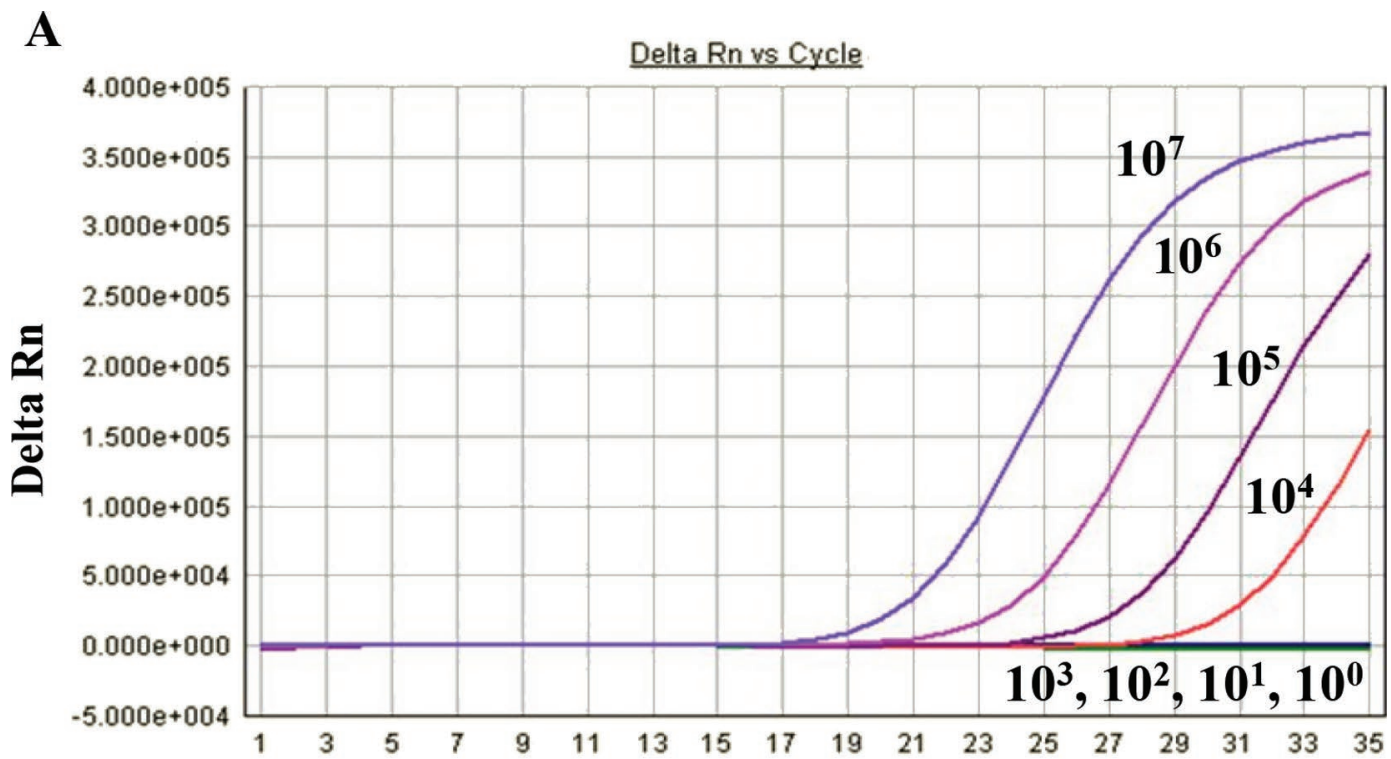

B

Cycle Number

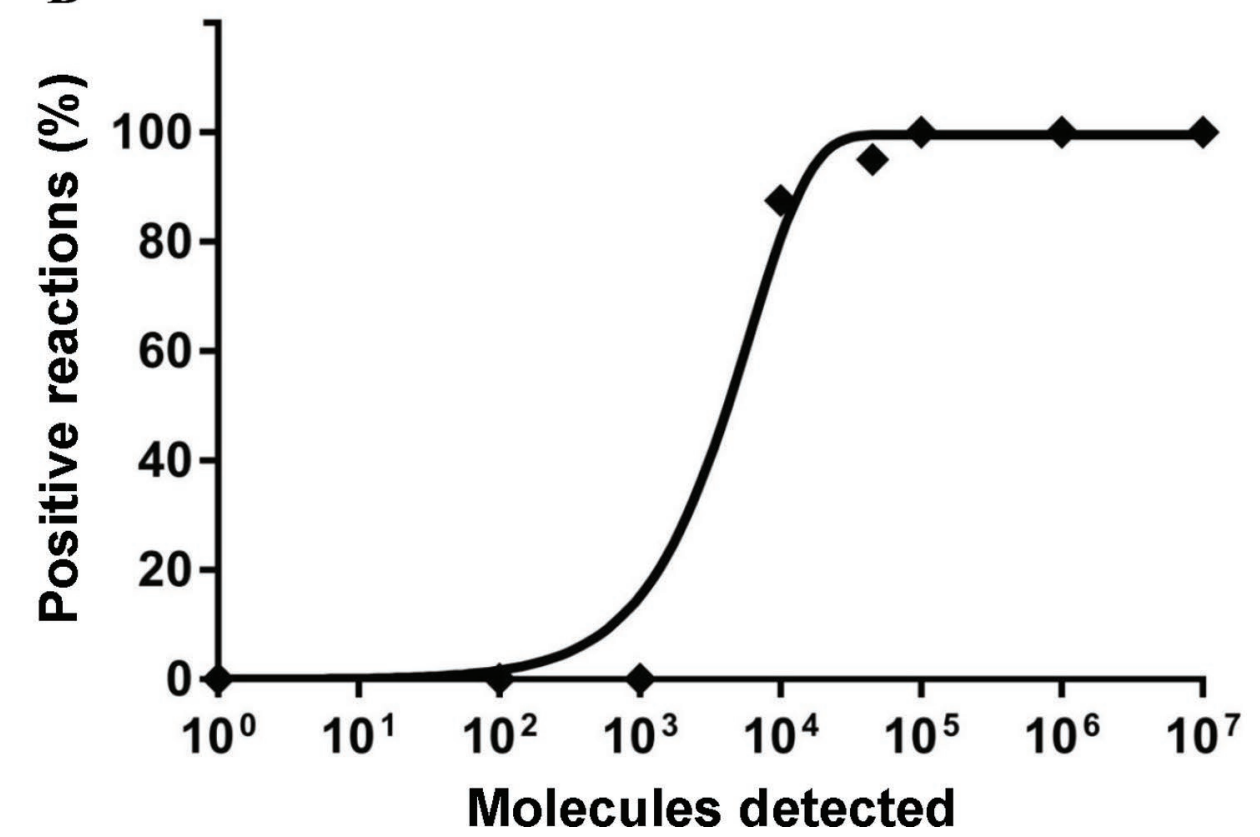

Figure 2. Analytical sensitivity of real-time PCR assay. (A) Real-time PCR had the same detection limit, $10^{4}$ copies/reaction. (B) Probit regression analysis based on the results of 8 runs using SPSS Statistics 22 software (IBM Corp., Armonk, NY). The limit of detection of $95 \%$ probability $\left(4.5 \times 10^{4}\right.$ copies $)$ of real-time PCR is indicated by a rhomboid. Color version available online. 
Table 3. Comparison of reaction time of different methods for Cronobacter spp. detection in contaminated powdered infant formula milk $(\mathrm{PIF})^{1}$

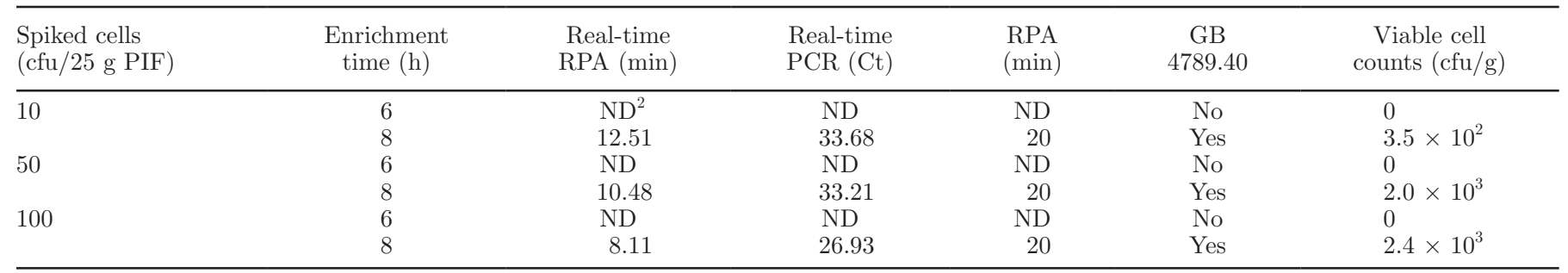

${ }^{1} \mathrm{RPA}=$ recombinase polymerase amplification; GB4789.40 = Chinese traditional detection method (CFDA, 2016).

${ }^{2} \mathrm{ND}=$ not detected.

Using the Cronobacter spp. plasmid DNA as the template, it was detected between 8 and 15 min for $10^{7}$ to $10^{4}$ copies of DNA. The LOD of real-time RPA was approximately $2.3 \times 10^{4}$ copies/reaction, which was quite similar to that of PRA and real-time PCR using the same diluted Cronobacter spp. DNA in our study.

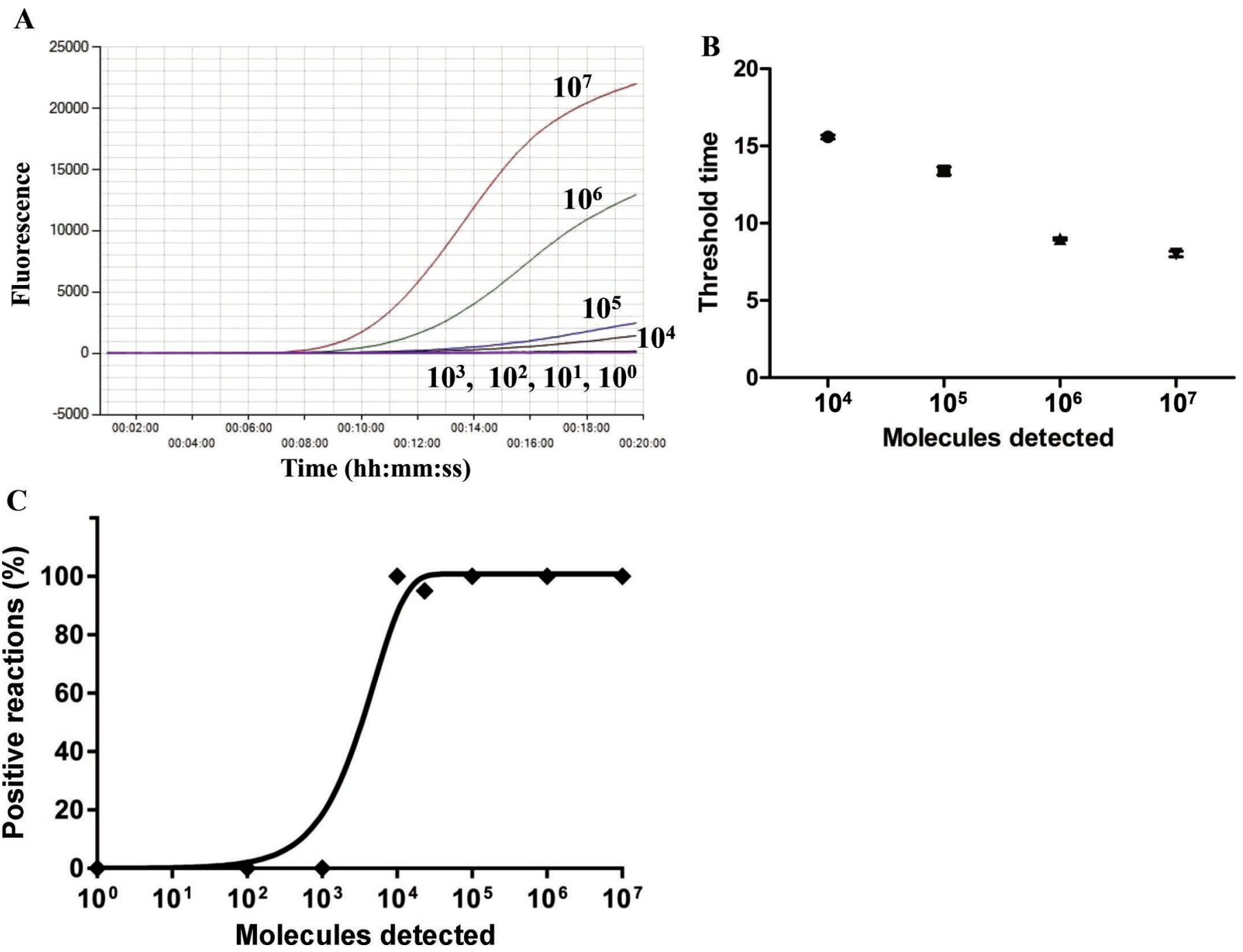

Figure 3. Performance of real-time recombinase polymerase amplification (RPA) for detection of Cronobacter spp. (A) Fluorescence development over time using a dilution range of $10^{7}$ to $10^{0}$ copies of the standard DNA. (B) Semilogarithmic regression of the data collected from 8 runs on the standard DNA using GraphPad Prism 7.0 (GraphPad Software Inc., San Diego, CA). The runtimes of real-time RPA were approximately 8 to $15 \mathrm{~min}$ for $10^{7}$ to $10^{4}$ copies. (C) A probit regression analysis. The limit of detection of the real-time RPA was approximately $2.3 \times 10^{4}$ copies/reaction in $95 \%$ of cases and indicated by a rhomboid. Color version available online. 
The detection rate of real-time RPA assay was comparable to that of real-time PCR, although the former assay we developed was much faster than the latter. In addition, the potential use of real-time RPA to detect Cronobacter spp. was demonstrated with artificially contaminated PIF samples. Our results showed that real-time RPA had a diagnostic agreement of $100 \%$ with real-time PCR and the traditional method.

Real-time RPA is a new pathogen detection technique with advantages over the traditional detection method and real-time PCR (Lillis et al., 2016; Wang et al., 2017a,b). Currently, RPA has been explored widely for molecular detection of diverse pathogens, and real field testing has also been achieved (Abd El Wahed et al., 2015a,b; Lillis et al., 2016). Furthermore, the portable point-of-care tube scanner (Genie III, OptiGene Limited) used in our study was much smaller, lighter, and cheaper than the real-time PCR machine and can be used in the field when run on battery power. Compared with DNA amplification methods, RPA assay has several advantages: (1) RPA does not require initial heating for DNA denaturation, which shortens the reaction time; (2) RPA shows a certain degree of tolerance to common PCR inhibitors; (3) RPA can tolerate 5 to 9 mismatches in the primer and probe without affecting the performance of the assay; and (4) RPA uses reagents stored in lyophilized pellets, which are stable and can react satisfactorily at $25^{\circ} \mathrm{C}$ for up to $12 \mathrm{wk}$ and at $45^{\circ} \mathrm{C}$ for up to $3 \mathrm{wk}$. These distinguishing features of RPA and hands-on experience of real field testing using RPA make us believe that RPA could work excellently in a clinical diagnostic setting; real-time RPA serves as an efficient tool for implementation in food for the detection of Cronobacter spp. in less-advanced laboratories and is suitable for field detection.

\section{CONCLUSIONS}

In the present study, our newly developed RPA and real-time RPA assays could be successfully used for sensitive and specific detection of Cronobacter spp. in contaminated PIF with significantly reduced time consumption. The analysis time was reduced to less than $15 \mathrm{~min}$, and the assays were as reliable as real-time PCR and the traditional method. All tests proved that the RPA and real-time RPA assays hold great promise for rapid, reliable, and sensitive detection of Cronobacter spp. in foods.

\section{ACKNOWLEDGMENTS}

This study was supported by the Program for the Natural Science Foundation of Hebei Province (C2017205129, Hebei, China), Young Top-notch Tal- ents in Universities of Hebei Province (BJ2016033, Hebei, China), China Postdoctoral Science Foundation (2017M621099), the Research Project of General Administration of Quality Supervision, Inspection and Quarantine of China (2016IK107), and the Doctoral Foundation of Hebei Normal University (L2016B13, Hebei, China).

\section{REFERENCES}

Abd El Wahed, A., P. Patel, O. Faye, S. Thaloengsok, D. Heidenreich, P. Matangkasombut, K. Manopwisedjaroen, A. Sakuntabhai, A. A. Sall, F. T. Hufert, and M. Weidmann. 2015a. Recombinase polymerase amplification assay for rapid diagnostics of dengue infection. PLoS One 10:e0129682.

Abd El Wahed, A., M. Weidmann, and F. T. Hufert. 2015b. Diagnostics-in-a-suitcase: Development of a portable and rapid assay for the detection of the emerging avian influenza A (H7N9) virus. J. Clin. Virol. 69:16-21.

Cai, X. Q., H. Q. Yu, Z. X. Ruan, L. L. Yang, J. S. Bai, D. Y. Qiu, Z. H. Jian, Y. Q. Xiao, J. Y. Yang, T. H. Le, and X. Q. Zhu. 2013. Rapid detection and simultaneous genotyping of Cronobacter spp. (formerly Enterobacter sakazakii) in powdered infant formula using real-time PCR and high resolution melting (HRM) analysis. PLoS One 8:e67082.

CFDA. 2016. GB4789.40-2016. National Food Safety Standard-Food Microbiological Examination-Examination of Cronobacter (Enterobacter sakazakii). China Food and Drug Administration, Beijing, China.

Chen, Q., Y. Li, T. Tao, X. Bie, F. Lu, and Z. Lu. 2017. Development and application of a sensitive, rapid, and reliable immunomagnetic separation-PCR detection method for Cronobacter spp. J. Dairy Sci. 100:961-969.

Drudy, D., N. R. Mullane, T. Quinn, P. G. Wall, and S. Fanning. 2006. Enterobacter sakazakii: An emerging pathogen in powdered infant formula. Clin. Infect. Dis. 42:996-1002.

FDA. 2002. Isolation and Enumeration of Enterobacter sakazakii from Dehydrated Infant Formula. United States Food and Drug Administration, Rockville, MD.

Hu, S., Y. Yu, R. Li, X. Wu, X. Xiao, and H. Wu. 2016. Rapid detection of Cronobacter sakazakii by real-time PCR based on the cgcA gene and Taqman probe with internal amplification control. Can. J. Microbiol. 62:191-200.

Hunter, C. J., and J. F. Bean. 2013. Cronobacter: An emerging opportunistic pathogen associated with neonatal meningitis, sepsis and necrotizing enterocolitis. J. Perinatol. 33:581-585.

ISO. 2006. ISO/TS 22964:2006. Milk and Milk Products-Detection of Enterobacter sakazakii. International Organization for Standardization, Geneva, Switzerland.

Jaradat, Z. W., Q. O. Ababneh, I. M. Saadoun, N. A. Samara, and A. M. Rashdan. 2009. Isolation of Cronobacter spp. (formerly Enterobacter sakazakii) from infant food, herbs and environmental samples and the subsequent identification and confirmation of the isolates using biochemical, chromogenic assays, PCR and $16 \mathrm{~s}$ rRNA sequencing. BMC Microbiol. 9:225.

Kandhai, M. C., M. W. Reij, L. G. Gorris, O. Guillaume-Gentil, and M. van Schothorst. 2004. Occurrence of Enterobacter sakazakii in food production environments and households. Lancet 363:39-40.

Li, Y., Q. Chen, H. Jiang, Y. Jiao, F. Lu, X. Bie, and Z. Lu. 2016. Novel development of a qPCR assay based on the rpoB gene for rapid detection of Cronobacter spp. Curr. Microbiol. 72:436-443.

Lillis, L., J. Siverson, A. Lee, J. Cantera, M. Parker, O. Piepenburg, D. A. Lehman, and D. S. Boyle. 2016. Factors influencing recombinase polymerase amplification (RPA) assay outcomes at point of care. Mol. Cell. Probes 30:74-78.

Muytjens, H. L., H. Roelofs-Willemse, and G. H. Jaspar. 1988. Quality of powdered substitutes for breast milk with regard to members of the family Enterobacteriaceae. J. Clin. Microbiol. 26:743-746. 
Park, S., S. Shukla, Y. Kim, S. Oh, S. Hun Kim, and M. Kim. 2012. Development of sandwich enzyme-linked immunosorbent assay for the detection of Cronobacter muytjensii (formerly called Enterobacter sakazakii). Microbiol. Immunol. 56:472-479.

Pina-Pérez, M. C., D. Rodrigo, and A. Martinez. 2016. Nonthermal inactivation of Cronobacter sakazakii in infant formula milk: A review. Crit. Rev. Food Sci. Nutr. 56:1620-1629.

Qiming, C., T. Tingting, B. Xiaomei, L. Yingjian, L. Fengxai, Z. Ligong, and L. Zhaoxin. 2015. Mining for sensitive and reliable species-specific primers for PCR for detection of Cronobacter sakazakii by a bioinformatics approach. J. Dairy Sci. 98:5091-5101.

Shukla, S., G. Lee, X. Song, J. H. Park, H. Cho, E. J. Lee, and M. Kim. 2016. Detection of Cronobacter sakazakii in powdered infant formula using an immunoliposome-based immunomagnetic concentration and separation assay. Sci. Rep. 6:34721.

van Acker, J., F. de Smet, G. Muyldermans, A. Bougatef, A. Naessens, and S. Lauwers. 2001. Outbreak of necrotizing enterocolitis associated with Enterobacter sakazakii in powdered milk formula. J. Clin. Microbiol. 39:293-297.
Wang, J. C., L. Liu, Q. Han, J. Wang, and W. Yuan. 2017b. An exo probe-based recombinase polymerase amplification assay for the rapid detection of porcine parvovirus. J. Virol. Methods 248:145147.

Wang, J., J. Wang, L. Liu, and W. Yuan. 2017a. Development of a real-time recombinase polymerase amplification assay for rapid and sensitive detection of porcine circovirus 2. Arch. Virol. 162:2293-2296.

Willis, J., and J. E. Robinson. 1988. Enterobacter sakazakii meningitis in neonates. Pediatr. Infect. Dis. J. 7:196-199.

Yu, S., L. Yan, X. Wu, F. Li, D. Wang, and H. Xu. 2017. Multiplex PCR coupled with propidium monoazide for the detection of viable Cronobacter sakazakii, Bacillus cereus, and Salmonella spp. in milk and milk products. J. Dairy Sci. 100:7874-7882.

Zhou, B., B. Chen, X. Wu, F. Li, P. Yu, Z. P. Aguilar, H. Wei, and H $\mathrm{Xu}$. 2016. A new application of a sodium deoxycholate-propidium monoazide-quantitative PCR assay for rapid and sensitive detection of viable Cronobacter sakazakii in powdered infant formula. J. Dairy Sci. 99:9550-9559. 